\title{
Characterization of wood micro-morphology from gas permeability measurements
}

Wei $\mathrm{AI}^{1}$, Hervé DUVAL $^{1 *}$, Floran PIERRE ${ }^{1}$ and Patrick PERRÉ ${ }^{1}$

${ }^{1}$ Laboratoire de Génie des Procédés et Matériaux, CentraleSupélec, Université Paris-Saclay, Châtenay-Malabry, 92295, France

*corresponding author, herve.duval@centralesupelec.fr, +33 141131731.

Abstract: This paper presents a method for identifying wood morphological parameters from gas apparentpermeability measurements. The apparent permeability at a given mean pressure is typically determined from the pressure relaxation kinetics when the gas permeates through the wood sample between two compartments which have different initial pressures. Using the proposed set-up apparent permeability values ranging from $10^{-10}$ to $10^{-18} \mathrm{~m}^{2}$ can be measured with a mean gas pressure varying from 2 bar down to 35 mbar. Morphological and topological parameters are then identified from the variations in apparent permeability as a function of the mean gas pressure using a pore network model. The network consists of elements such as pipes or orifices connected in series or in parallel. The rarefied gas flow is described in each element by an appropriate model and the unknowns are determined by an inverse method.

This approach was first applied to track-etched polycarbonate membranes for validation purposes. The calculated pore radius and pore density values were compared to observations by environmental scanning electron microscopy. Softwood specimens were then investigated. The mean radius of the pores controlling permeability in the longitudinal and tangential directions was determined and found to be in good agreement with literature data.

Keywords: porous media, cellular tissue, pore network model, rarefied gas dynamics, slip flow.

Acknowledgements: We are grateful to J. Casalinho, T. Martin and J. Trubuil for their contribution in designing and building the set-up and to N. Ruscassier for the ESEM observations and for the micrographs presented in this article. 


\section{Introduction}

Wood is a fine example of a natural microfluidic system. In conifers (softwood trees), sap is transported through microscale elongated cells called tracheids, which run lengthwise along the trunk and are interconnected via small valves called bordered pits (see Fig. 1a-d). The high aspect ratio of tracheids facilitates sap circulation. According to the cohesion-tension theory (Dixon and Joly 1894), rising sap is driven by capillarity and evaporation from leaves. The sap pressure in the tree is thus significantly lower than atmospheric pressure and is even negative since most trees are more than $10 \mathrm{~m}$ high (Scholander et al. 1965). In the vascular system of trees, the bordered pits not only ensure the connection between the tracheids but also prevent the living tree from embolism in the event of air intrusion (as a result of injury or cavitation for example). So if a gas/liquid interface passes through the pit, the capillary forces cause the flap of the valve to move into the so-called aspired position, closing off the gas flow from embolized (gas-filled) tracheids to adjacent water-filled tracheids. As shown in Fig. 1e-f, the valve consists of a disc-shaped membrane, or torus, attached to the pit chamber by microfibrillar strands forming the so-called annular margo. The openings between the strands allow the sap to pass through when the pit is non-aspired. Conversely, when the pit is aspired, the torus is pressed against the pit aperture on the waterfilled side of the bordered pit (see the electron micrographs of Comstock and Côte (1968)). When green wood is cut and naturally dried, many bordered pits are aspired as a consequence of the "anti-embolism" mechanism. Due to the wood structure, pits are more easily aspired in earlywood, formed during spring, than in latewood, formed during summer (Phillips 1933, Almeida et al. 2008). A scanning electron micrograph of a transverse section of softwood (Fig. 1b) shows a quite sudden transition between earlywood and latewood. In latewood, the cell walls are thicker and the radial extension of tracheids is much smaller. The book by Siau (1995) gives more details on wood anatomy and physics.
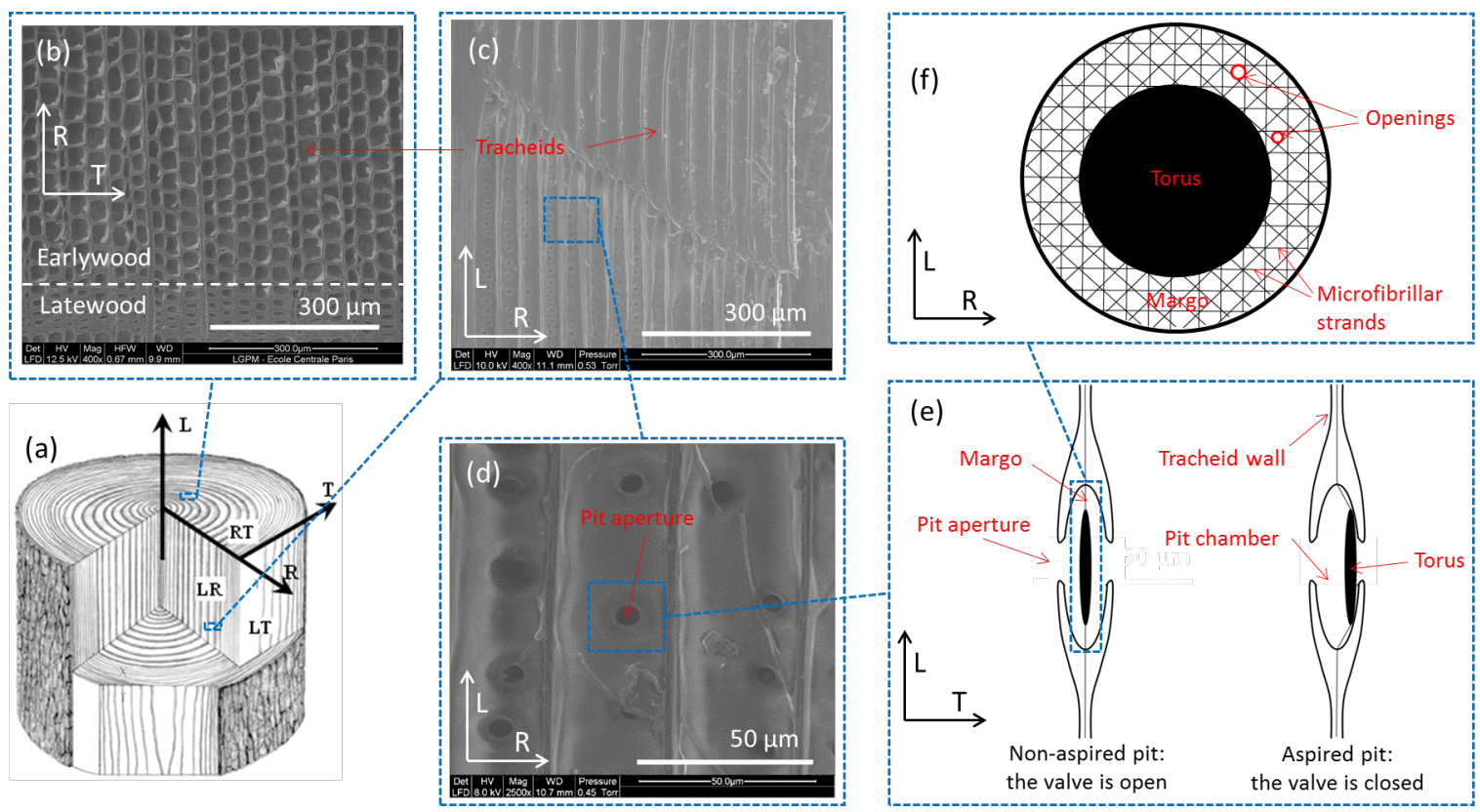

Fig. 1 Multiscale structure of softwood tissues: (a) the main directions of a wood log, (b) micrograph of Norway spruce tracheids by ESEM in RT plane, (c) micrograph of Norway spruce tracheids by ESEM in LR plane, (d) micrograph of bordered pits by ESEM in LR plane, (e) diagram of a bordered pit in LT plane in open and closed positions, (f) diagram of the openings in the margo in LR plane, the openings are characterized by the diameter of the circle inscribed between the bordering microfibrils

In dry wood, transport properties depend mainly on the tracheid network, especially on the conductive (i.e. nonaspired) bordered pits. These properties govern the heat and moisture transfers involved in many wood transformation processes and uses. In order to better understand, predict and control these phenomena, a detailed knowledge of wood multiscale morphology is required. The pore-size ranges from about $10 \mu \mathrm{m}$ for the tracheid lumen down to $0.1 \mu \mathrm{m}$ for the margo openings. In the present paper, the term "micro-morphology" refers to this size-range. In this sense, we follow the life science classification (ASTM F2450-10 2010) which defines micropores as pores having diameter between $0.1 \mu \mathrm{m}$ and $100 \mu \mathrm{m}$. This pore classification is more appropriate for wood tissue characterization than IUPAC recommendations (Rouquerol et al. 1994) which define micropores 
as pores having diameter smaller than $2 \mathrm{~nm}$. Wood is usually characterized by serial cross-section analysis or micro-tomography. However, submicrometer-sized morphological features such as openings between microfibrillar strands are difficult, if not impossible, to assess using such methods.

An alternative approach developed by Petty and coworkers is based on an analysis of apparent-permeability variations as a function of gas pressure (Petty and Preston 1969, Petty 1970). This approach involves using flow regime changes (from viscous flow to slip flow for example), which occur at different mean pressures depending on pore size, to identify pore-network characteristics.

A detailed development of this concept starts with Darcy's law which relates the volume flow rate $Q$ to the pressure gradient:

$$
Q=-\frac{K_{\mathrm{app}} A}{\mu} \frac{d P}{d x}
$$

where $\mu$ is the gas dynamic viscosity, $A$ is the porous medium cross-sectional area and $d P / d x$ is the pressure gradient in the flow direction $x . K_{\text {app }}$ is the so-called apparent permeability which coincides with the intrinsic permeability in the viscous regime, i.e. when the gas mean free path, $\lambda$, is small compared to the characteristic pore radius $r$ of the medium. For a hard sphere gas approximation, $\lambda$ is given by (Hirschfelder et al. 1954):

$$
\lambda=\frac{16 \mu}{5 P} \sqrt{\frac{R T}{2 \pi M}}
$$

where $R$ is the ideal gas constant, $M$ the molar mass of the gas and $T$ its temperature. The density of the gas phase is given by $\rho=P M / R T$. When the Knudsen number, $\mathrm{Kn}=\lambda / r$, increases (typically from $10^{-2}$ or more), gas slippage in the pores increases, leading to apparent permeability values higher than the intrinsic permeability.

Petty's approach consists in measuring the volumetric flow rate of gas permeating through a porous medium sample. The sample is connected upstream to an expansion valve and a chamber maintained at atmospheric pressure and downstream to a vacuum reservoir. Tests are carried out at a given initial pressure difference across the sample but at various initial downstream pressures. Gas volumetric flow rates are measured at atmospheric pressure upstream of the expansion valve. Strictly speaking, the gas flow is transient during a test since the setup behaves as a closed system. However, it is usually possible to define a time interval during which the gas flow is quasi-steady and to perform the measurements during that period.

The measurements were analyzed on the basis of Adzumi's experiments (Adzumi 1937). In the simplest approach (Sebastian et al. 1965), the hydraulic resistance of the tracheids was neglected and the conductive pits were assumed to control wood permeability. The hydraulic conductance of a conductive pit depends on the number and diameter of openings in the margo of the bordered pit. In that which follows, the terms "resistance" and "conductance" will implicitly refer to the hydraulic resistance and to the hydraulic conductance, respectively, and the shortened expression "pit opening" will refer to an opening in the margo of a bordered pit. The pit opening network was modelled by identical cylindrical pores connected in parallel (inside a tracheid) and in series (between tracheids). Using Adzumi's equation established for a single pore, the gas volumetric flow rate passing through a sample can then be expressed as:

$$
Q_{a}=\frac{N \pi r^{4} P_{m} A \Delta P}{8 \mu P_{a} L_{c}}+\varphi \frac{4}{3} \sqrt{\frac{2 \pi R T}{M}} \frac{N r^{3} A \Delta P}{P_{a} L_{c}}
$$

where $Q_{a}$ is measured at pressure $P_{a}$ (in Petty's set-up, $P_{a}$ is atmospheric pressure), $\Delta P$ is the pressure difference across the sample, $P_{m}$ is the mean pressure in the sample, $r$ is the effective pit opening radius and $N$ is the number of conductive pit openings per unit cross-sectional area. $L_{c}$ is the cumulative pore length estimated from the margo thickness and the sample dimensions. $\varphi$ is a coefficient depending on the Knudsen number.

Adzumi's experiments showed that for a single gas, $\varphi$ varies between 1 and 0.9 depending on the gas pressure (Adzumi 1937). Adzumi's equation is thus essentially the superposition of two flows, i.e, a viscous flow (first term of the right-hand side of Eq. 3) and a Knudsen flow (second term of the right-hand side of Eq. 3 weighted by $\varphi$ ). Sebastian et al. (1965) applied their model to white spruce wood: experimental data were represented in a so-called Adzumi plot, i.e., $Q_{a} P_{a} L / \Delta P$ as a function of $P_{m}$. Plots were linear in accordance with Eq. 3. The 
effective radius of the pit openings $r$ and the number of conductive pit openings per area unit $N$ can be deduced from the intercept and slope of the curve. However, in many softwood species, the plots are found to be curvilinear rather than linear. Petty and coworkers explained this behavior by the low but non-negligible resistance of the tracheids coupled with the high resistance of the pit openings. They therefore extended Sebastian's approach to take into account both contributions and were able determine not only the pit opening radius and the number of conductive pit openings per unit cross-sectional area but also the tracheid radius and the number of conductive tracheids per unit cross-sectional area.

This characterization method was extensively used by Petty and coworkers, mostly in the seventies (see the references in the article of $\mathrm{Lu}$ and Avramidis 1999). This method made a significant contribution to understanding softwood permeability and determining the conductive pit openings as the limiting step along the flow pathway.

Gas permeability measurement and analysis are also an important and recurrent topic in petroleum and geophysical engineering. Klinkenberg (1941) first studied the effect of gas slippage in porous media (also known as the Klinkenberg effect) and proposed a correction for apparent permeability with respect to intrinsic permeability:

$$
K_{\text {app }}=K_{\infty}\left(1+\frac{b_{k}}{P_{m}}\right)=K_{\infty}(1+4 c \mathrm{Kn})
$$

where $b_{k}$ is the Klinkenberg slip coefficient and $c$ a factor close to 1 . It should be noted that revising Adzumi's equation leads to a similar relation with $c=\varphi / 3$. The original issue was to estimate the intrinsic permeability or liquid equivalent permeability of porous rocks from gas permeability measurements (Klinkenberg 1941, Florence et al. 2007). Another issue has recently come to light concerning the extraction of hydrocarbon gases from unconventional gas reservoirs such as shale-gas and coal-bed methane reservoirs. This has given rise to numerous modeling studies of gas flow in tight porous media (Florence et al. 2007, Civan 2010). These studies benefit from the advances made in rarefied gas theory over the last fifty years and of its more recent development in the context of micro- and nano-fluidics.

The different flow regimes that can be met in micro- and nano-fluidics, and the associated modeling approaches are summarized below. Depending on the gas rarefaction level, four flow regimes may be distinguished (Beskok et al. 1996): continuum flow for $\mathrm{Kn} \leq 10^{-3}$, slip flow for $10^{-3} \leq \mathrm{Kn} \leq 10^{-1}$, transition flow for $10^{-1} \leq \mathrm{Kn} \leq$ 10 and free molecular flow for $\mathrm{Kn} \geq 10$. For $\mathrm{Kn} \leq 10^{-3}$, the gas flow is adequately described by the NavierStokes equations coupled with a no-slip boundary condition at the solid surfaces. Note that the viscous flow regime (or Darcy regime) corresponds to the specific case of continuum flow with negligible inertial effects (i.e. when the Reynolds number based on the intrinsic permeability $\operatorname{Re}_{K}=\rho(Q / A) \sqrt{K_{\infty}} / \mu$ is typically lower than $10^{-2}$ ). In the latter case, Eq. 1 applies and $K_{\text {app }}=K_{\infty}$. For $10^{-3} \leq \mathrm{Kn} \leq 10^{-1}$, the Navier-Stokes equations must be coupled with suitable slip boundary conditions at the solid surfaces. The use of the first order Maxwell slip boundary condition (Maxwell 1879) leads to the above-mentioned Klinkenberg correction (Eq. 4). For Kn $\geq$ $10^{-1}$, Navier-Stokes equations break down and must be substituted either by a higher-order continuum approximation (such as the Burnett equation) or by the Boltzmann transport equation. The latter case corresponds to the so-called kinetic approach where the gas is described in terms of a velocity distribution function. The Boltzmann equation can be directly simulated using appropriate numerical methods such as the Direct Simulation Monte Carlo (DSMC) method (Bird 1996, Karniadakis et al. 2005). Alternative methods based on gas kinetic theory have also been developed (see for instance the model of Zhu and Ye (2010) inspired by the Mott-Smith moment method or the multiple temperature kinetic model of Liu et al. (2012)). More details on the various models developed in the different flow regimes can be found in the articles of Sharipov and Seleznev (1998) and Zhang et al. (2012).

Since Klinkenberg's correction is only valid in the slip flow regime, several authors have proposed new models to extend the validity of the permeability correction to higher Knudsen numbers. Civan's work (2010) based on Beskok and Karniadakis's equation for volumetric gas flow through a single pipe (1999) is worthy of particular mention. Beskok and Karniadakis's model is a physical-based empirical model applicable for the entire Knudsen number range. It has been validated by comparing its results with DSMC simulation results and experimental data. More recently, Lv et al. (2014) applied Brenner's theory of bivelocity hydrodynamics (2005) to rarefied gas flows in micro- and nano-tubes and obtained a permeability correction that is also valid over the entire Knudsen number range. 
In the present study, an innovative in-house experimental apparatus was developed that is capable of measuring gas permeability of porous media over a wide range of permeability values (from $10^{-10}$ to $10^{-18} \mathrm{~m}^{2}$ ) and a wide range of mean gas pressures (from 2 bar down to 35 mbar). Measurements rely on accurate pressure sensors instead of gas flowmeters as in standard methods. The transient character of the experiments is rigorously taken into account in the data analysis and the apparent permeability is determined from the relaxation kinetics of the pressure difference across the tested sample. The estimated permeability can de determined either under an isothermal assumption or without this simplifying assumption depending on the characteristic pressure relaxation time. The morphological parameters are then determined from variations in the apparent permeability as a function the reciprocal mean pressure using a pore network model.

The experimental set-up is described in the next section. Details are then given of the measurements taken on well-defined porous materials, i.e. polycarbonate track-etched membranes, and on a well-documented softwood species, i.e. Norway spruce. Finally, the identification approach is presented and applied to our experimental datasets and the values of the identified morphological parameters are compared to microscopic observations and to data found in literature.

\section{Materials and methods}

\subsection{Sampling}

Specimens of ultrafiltration membranes and Norway spruce (Picea abies) were used in this study.

Commercial track-etched polycarbonate membranes were used $(80 \mathrm{~nm}$ and $10 \mu \mathrm{m}$ Whatman Nuclepore membranes, GE Healthcare Life Sciences), purchased from Fisher Scientific. These membranes are symmetrical and have controlled cylindrical pores. For the $80 \mathrm{~nm}($ resp. $10 \mu \mathrm{m}$ ) track-etched membranes, the nominal pore radius is equal to $40 \mathrm{~nm}$ (resp. $5 \mu \mathrm{m}$ ), the nominal mean pore density is equal to $6 \times 10^{8}$ pores $/ \mathrm{cm}^{2}$ (resp. $10^{5}$ pores $/ \mathrm{cm}^{2}$ ) and the nominal membrane thickness is equal to $6 \mu \mathrm{m}$ (resp. $10 \mu \mathrm{m}$ ) as specified by the manufacturer. For the $80 \mathrm{~nm}$ (resp. $10 \mu \mathrm{m}$ ) membranes, disc samples with a diameter of $37 \mathrm{~mm}$ (resp. $8 \mathrm{~mm}$ ) were cut and used for permeability measurements.

Wood samples were prepared from a plank of Norway spruce with narrow and even annual rings (resonance wood quality). The plank (mature wood taken from the heartwood zone of the log) comes from a tree cut in the Risoux forest (Jura mountains, eastern France). Spruce specimens were dried under natural conditions. The material tested had a basic wood density (oven-dry mass to green volume) of $360 \mathrm{~kg} \cdot \mathrm{m}^{-3}$.

Due to the high aspect ratio of the wood cells, wood is a strongly anisotropic material and three material directions emerge, i.e. longitudinal, radial and tangential directions (see Fig. 1). In the present study, samples were taken in the longitudinal and tangential directions of the wood log. Two samples were prepared per direction. They are denoted L1 and L2 (resp. T1 and T2) in the longitudinal (resp. tangential) direction. The shape of the samples is adapted to the wood permeability in the corresponding direction. Hence in the longitudinal direction, characterized by high permeability values, $18 \mathrm{~mm}$ diameter, $30 \mathrm{~mm}$ thick rod samples were cut. In the tangential direction, characterized by low permeability values, $72 \mathrm{~mm}$ diameter, $10 \mathrm{~mm}$ thick disc samples were cut. In order to avoid lateral leakage during permeability measurements, an impervious contact surface with the rubber tube is required (see the experimental apparatus section). This is obtained by coating the lateral surface of the sample with two successive layers of epoxy resin.

\subsection{ESEM characterization}

The track-etched membranes were observed with an environmental scanning electron microscopy (ESEM, FEI Quanta 200) in high vacuum mode after the deposition of a nanometer thick gold film (using EMITECH K550 coating system). The micrographs (see an example in Fig. 2) were analyzed using the public domain software ImageJ (Rasband 2014) to determine the mean pore radius and the mean pore density of the membranes. For the $80 \mathrm{~nm}($ resp. $10 \mu \mathrm{m})$ membranes, the measured mean pore radius is equal to $39 \mathrm{~nm}$ (resp. $4.4 \mu \mathrm{m}$ ) with a standard deviation of $5 \mathrm{~nm}$ (resp. $0.5 \mu \mathrm{m}$ ). The measured mean pore density is equal to $5.9 \pm 0.7 \times 10^{8}$ pores $/ \mathrm{cm}^{2}$ (resp. $8.6 \pm 0.1 \times 10^{4}$ pores $/ \mathrm{cm}^{2}$ ). The measured membrane thickness is equal to $4.2 \pm 0.5 \mu \mathrm{m}$ (resp. $9.8 \pm 1.4 \mu \mathrm{m})$. These values are consistent with the manufacturer's specifications. 


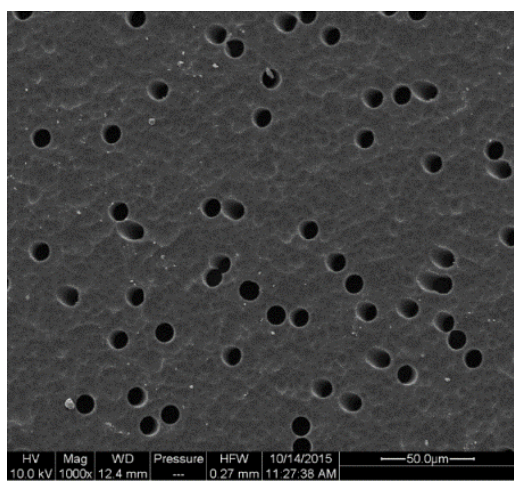

Fig. 2 Micrograph of a $10 \mu \mathrm{m}$ track-etched membrane (Whatman Nuclepore) by ESEM. The black disks correspond to membrane pores.

The surfaces of the Norway spruce specimens were carefully cut using a sledge microtome after a two-day immersion in distilled water. They were then dried under natural conditions before being observed by ESEM in low vacuum mode. Typical micrographs of radial and transverse sections are shown in Fig. 1. The tracheid dimensions on transverse sections were measured. In earlywood, the mean tracheid radial dimension $(w=$ $44.5 \pm 4.7 \mu \mathrm{m})$ is slightly greater than the mean tracheid tangential dimension $(l=30.1 \pm 6.2 \mu \mathrm{m})$ and the equivalent radius (pipe radius keeping the same cross-sectional area) of the tracheid lumen is equal to $14.8 \mu \mathrm{m}$ with a standard deviation of $1.8 \mu \mathrm{m}$. In latewood, the mean tracheid tangential dimension $(l=30.6+4.6 \mu \mathrm{m})$ is about the same as in earlywood, simply because they are produced by the same mother cells, but the mean tracheid radial dimension is significantly lower, i.e. $w=17.0 \pm 3.3 \mu \mathrm{m}$. Furthermore, the cell walls are thicker. Consequently, the equivalent radius of the tracheid lumen in latewood is narrower and equal to $5.1 \mu \mathrm{m}$ with a standard deviation of $1.9 \mu \mathrm{m}$. Lastly, the mean aperture radius of the bordered pits is equal to $3.0 \mu \mathrm{m}$ with a standard deviation of $0.3 \mu \mathrm{m}$. These values are in agreement with the data reported by Brändström for Norway spruce (2001).

\subsection{Experimental apparatus}

An in-house device was designed to measure the apparent gas permeability over a wide range of permeability values and average pressures. The measurement principle consists in imposing a pressure difference across the tested sample and calculating its permeability by determining the flow rate from pressure relaxation kinetics. The apparatus and the experimental protocol are described in detail in another paper (Ai et al. 2017). Consequently, only a brief outline will be given here.

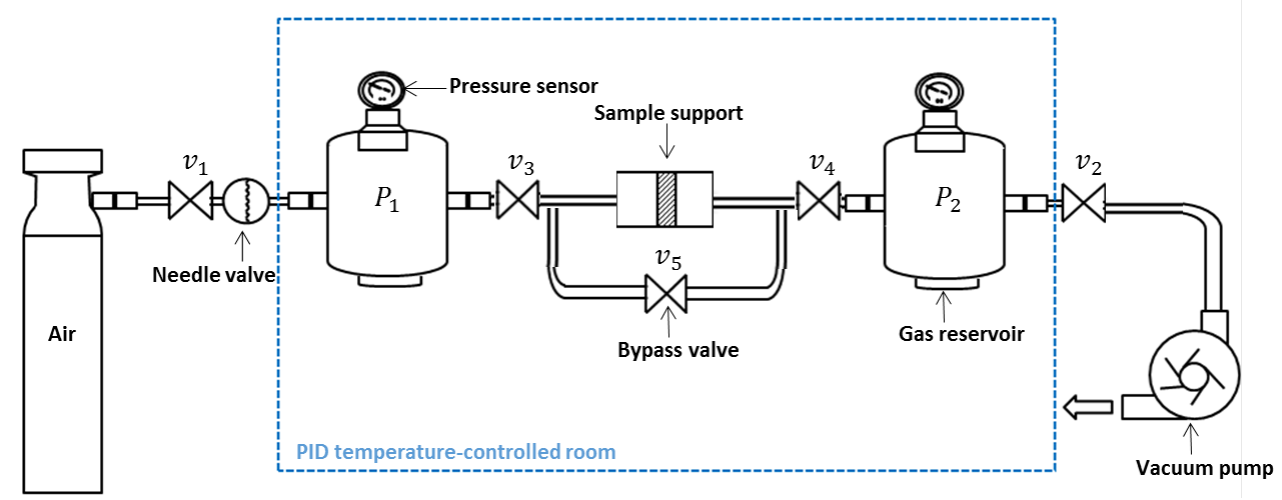

Fig. 3 Experimental device designed to measure the apparent gas permeability of wood samples. The sample support (middle) is built in series with two rigid tanks. The tank on the left is also connected to a compressed gas cylinder whereas the tank on the right is connected to a vacuum pump. Gas thus permeates from left to right.

As shown in Fig. 3, the device is composed of two rigid tanks of identical volume placed in series with a sample support. The high pressure tank (characterized by pressure $P_{1}$ ) is also connected to a compressed gas cylinder whereas the low pressure tank $\left(P_{2}\right)$ is connected to a vacuum pump. Each component of the system is separated by vacuum slide valves. The gas pressure in the tanks is measured by highly sensitive pressure sensors (GE 
Druck, TERPS RPS/DPS 8000). The sensors are capable of measuring pressures ranging from 0 to $2000 \mathrm{mbar}$ with a precision of $0.2 \mathrm{mbar}$ between $35 \mathrm{mbar}$ and $2000 \mathrm{mbar}$ at $25{ }^{\circ} \mathrm{C}$. The device is completely airtight. Furthermore, it is placed in a temperature-controlled chamber (accuracy $\pm 0.5^{\circ} \mathrm{C}$, standard deviation $0.04^{\circ} \mathrm{C}$ ). For the tests reported in the present paper, the temperature of the chamber was set at $T_{0}=30^{\circ} \mathrm{C}$.

Two sets of tanks $(V=0.2 \mathrm{~L}$ and $V=24 \mathrm{~L})$ are available as well as three sample supports compatible with three sample geometries, i.e. rod or disc for wood specimens and sheet for track-etched membranes. The tank volume and the sample geometry (for the wood specimens) are selected according to the expected sample permeability: rod shape and big tanks (resp. disk shape and small tanks) for highly (resp. slightly) permeable materials.

The sample supports are based on the ALU-CHA system developed by Agoua and Perre (2010). This system is essentially a tube-in-tube system with an inner rubber tube mounted coaxially in a hollow aluminum shell. The shell side can be connected either to a pump or to a compressed air system. The sample is inserted in the rubber tube after pumping the air from the shell. If the sample is rigid, it is inserted as is, otherwise it is first mounted on a rigid frame. An overpressure of more than 2 bar is then applied at the shell side to ensure air tightness around the sample during measurements.

The intrinsic leak rate of this set-up was measured at the lowest experimental mean pressure (the worst conditions) using a PVC (polyvinylchloride) impermeable sample: the leak rate is $0.2 \mathrm{mbar} / \mathrm{h}$ at a mean pressure of $30 \mathrm{mbar}$ for the small tanks and $0.7 \mathrm{mbar} / \mathrm{h}$ at a mean pressure of $30 \mathrm{mbar}$ for the big tanks. These values are much lower than the pressure relaxation rates typically measured in the present study.

The measurement protocol begins by setting the two tanks at similar but different pressures $\left(P_{1}>P_{2}\right)$. At this stage, valves $v_{3}$ and $v_{4}$ are closed. Small initial pressure differences $\Delta P=P_{1}-P_{2}$ of a few mbar are preferred. However, for slightly permeable materials, long measurement times are required and a $\Delta P$ value of up to 100 mbar may be necessary for the "intrinsic" leak rate of the set-up to remain negligible compared to the measured pressure relaxation rates. Then, once thermal equilibrium has been reached, $v_{3}$ and $v_{4}$ are opened and the gas is allowed to permeate through the sample from the high pressure tank to the low pressure tank. The pressure variations in both tanks are recorded as a function of time. Experiments are carried out at various initial mean pressures $P_{m}=\left(P_{1}+P_{2}\right) / 2$.

\section{Results}

\subsection{Pressure relaxation}

A typical example of time-dependent pressure relaxation curves is shown in Fig. 4. The experimental data correspond to Norway spruce samples taken in the tangential direction of the wood log. It can be seen that the pressures of the high- and low-pressure tanks, i.e. $P_{1}$ and $P_{2}$ respectively, relax symmetrically toward the mean pressure $P_{m}=\left(P_{1}+P_{2}\right) / 2$ since the gas tanks have the same volume.

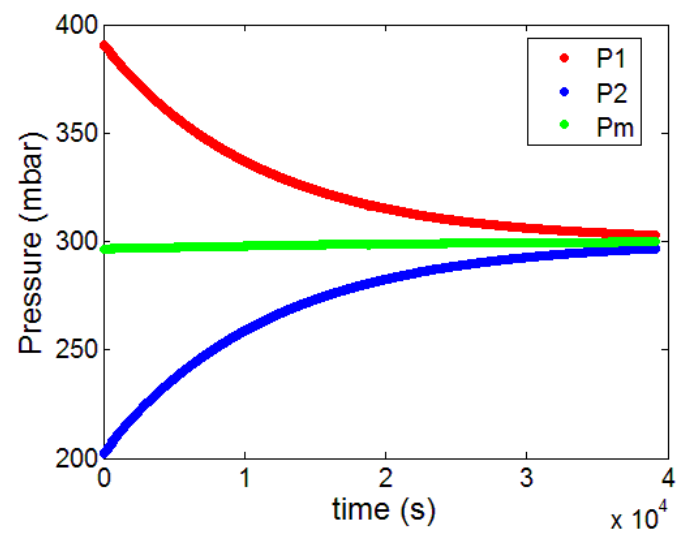

Fig. 4 Pressure relaxation kinetics in the high- and low-pressure tanks $\left(P_{1}\right.$ and $P_{2}$, respectively) for a Norway spruce sample taken in the tangential direction of the wood log at an initial mean pressure $P_{m}(0)=299$ mbar. $P_{1}$ and $P_{2}$ relax asymptotically toward the mean pressure $P_{m}(t)=\left(P_{1}(t)+P_{2}(t)\right) / 2$. 
The duration of an experiment may vary by several orders of magnitude, i.e. from a few seconds for the $10 \mu \mathrm{m}$ track-etched membranes to about $10^{5} \mathrm{~s}$ for a Norway spruce sample taken in the tangential direction of the wood log. In Fig. 4, the pressure relaxation through the sample is almost complete. Although complete relaxation is not necessary in order to determine the apparent permeability, these experimental curves have an exponential form. It can also be noted that the mean pressure "drift" remains very small compared to the pressure relaxation, thus confirming the low leak rate of the experimental set-up. In the rest of the article, no distinction will be made between the initial mean pressure $P_{m}(0)$ and the instantaneous value $P_{m}(t)$.

The rescaled pressure $\left(P_{1}-P_{m}\right) /\left(\left(P_{1}(0)-P_{2}(0)\right) / 2\right)$ was systematically plotted in semi-log graph form as a function of time (see examples on Fig. A1 and A2 in the electronic supplementary material) and the pressure relaxation was in fact found to be exponential for Norway spruce samples taken in the longitudinal and tangential directions. For the $80 \mathrm{~nm}$ and $10 \mu \mathrm{m}$ track-etched membranes, the relaxation deviates significantly from exponential behavior at high mean pressure.

A good estimate of the pressure relaxation characteristic time is given by:

$$
\tau_{R}=\left.\frac{P_{2}-P_{1}}{d\left(P_{1}-P_{2}\right) / d t}\right|_{t=0^{+}}
$$

If pressure relaxation in the tanks is rigorously exponential, then Eq. 5 in fact gives the exact characteristic time of the exponential law. For cases where the pressure relaxation is not exponential, then Eq. 5 gives an estimate of the short-term pressure relaxation characteristic time. This estimate was found to be lower than the long-term pressure relaxation characteristic time (see Fig. A1 in the electronic supplementary material).

For the experiments carried out on the $80 \mathrm{~nm}$ (resp. $10 \mu \mathrm{m}$ ) track-etched membranes with the big tanks, the pressure relaxation time $\tau_{R}$ varies from about $60 \mathrm{~s}$ at 30 mbar down to about $40 \mathrm{~s}$ at 2000 mbar (resp. from $30 \mathrm{~s}$ at $30 \mathrm{mbar}$ down to $2 \mathrm{~s}$ at $1000 \mathrm{mbar}$ ). For Norway spruce samples taken in the longitudinal (resp. tangential) direction with the big (resp. small) tanks, the pressure relaxation time varies from $2 \times 10^{4} \mathrm{~s}$ at 30 mbar down to $8 \times 10^{3} \mathrm{~s}$ at $1000 \mathrm{mbar}$ (resp. from $10^{4} \mathrm{~s}$ at $200 \mathrm{mbar}$ down to $7 \times 10^{3} \mathrm{~s}$ at $1000 \mathrm{mbar}$ ). It was found that the relaxation time increases for decreasing pressure values. This result appears to be rather counter intuitive since the slip effect increases for decreasing pressure values leading to higher apparent permeability: this point will be discussed later. The characteristic time of the heat transfer between the gas and the tank wall may be (over)estimated by the gas conduction time $\tau_{\mathrm{cd}}=v / L_{T}^{2}$ where $v$ is the kinematic viscosity of the gas at the corresponding pressure and $L_{T}$ the tank characteristic dimension. For the big (resp. small) tanks, $\tau_{\mathrm{cd}}$ varies from $50 \mathrm{~s}$ at $35 \mathrm{mbar}$ up to $1.5 \times 10^{3} \mathrm{~s}$ at $1000 \mathrm{mbar}$ (resp. from $2 \mathrm{~s}$ up to $60 \mathrm{~s}$ ). It can therefore be deduced that, for the measurements performed on Norway spruce samples (both directions), the variation in gas pressure in the tanks is essentially isothermal at $T_{0}$ at the experiment timescale. Conversely, for the measurements performed on the track-etched membranes, the variation in gas pressure in the tanks is expected to be non-isothermal at the experiment timescale. In that case, the tank walls may be considered to be isothermal at $T_{0}$ since their thermal inertia is very high compared to the inertia of the gas enclosed in the set-up.

\subsection{Determination of apparent permeability}

Consider a pressure relaxation test at the mean pressure $P_{m}$. If it is assumed that (i) the pressure drop $\left(P_{1}(t)-\right.$ $P_{2}(t)$ ) across the sample is small compared to the mean pressure $P_{m}$, (ii) the gas flow is spatially isothermal at $T(t)$ and (iii) the temperature difference $\left(T(t)-T_{0}\right.$ ) between the gas and the chamber remains small compared to the chamber temperature $T_{0}$, then Eq. 1 would be expected to be applicable and the apparent permeability would depend only on the material structure and on the Knudsen number of the gas flow in the medium. According to assumptions (i-iii), the Knudsen number is approximately constant with time and $K_{\text {app }}$ does not therefore vary during an experiment. Since the gas composition and the chamber temperature are identical from one test to another, $K_{\mathrm{app}}$ only depends on the gas mean pressure, for a given material and for a given mean flow direction. Last, a quasi-steady flow in the sample is considered (the gas residence time in the sample is typically much lower than the characteristic time $\tau_{R}$ ). Expressing the gas volumetric flow rate $Q(x, t)$ as a function of the molar flow $J(t)$, the temperature $T(t)$ and the pressure $P(x, t)$ and integrating Eq. 1 over the sample thickness $L$ leads to: 


$$
J(t)=\frac{K_{\mathrm{app}} A\left(P_{1}^{2}(t)-P_{2}^{2}(t)\right)}{2 \mu R T(t) L}
$$

Two methods are used to determine $K_{\text {app }}$ at $P_{m}$ and $T_{0}$. The first corresponds to the simplest case where the relaxation of the gas pressure in the tanks is rigorously isothermal and the second to the more general case where the relaxation is (slightly) non-isothermal.

\subsubsection{Isothermal regime}

If the pressure relaxation in the tanks is slow enough $\left(\tau_{R} \gg \tau_{\mathrm{cd}}\right)$, it may be assumed that the gas temperature always remains very close to the set-point temperature $T_{0}$ of the chamber. This corresponds typically to Norway spruce samples taken in the longitudinal and tangential directions of the wood log. The gas volumetric flow rate entering the sample can then be expressed as follows:

$$
Q(t)=V \frac{d P_{1} / d t}{P_{1}(t)}
$$

Since the tanks are of identical volume, the conservation of the gas enclosed in the set-up gives:

$$
\frac{d P_{1}}{d t}=-\frac{d P_{2}}{d t}
$$

The pressure relaxation rates in the tanks are equal but opposite. By combining Eq. 6 to 8 the time-dependent pressure difference variations across the sample are obtained:

$$
\frac{P_{1}(t)-P_{2}(t)}{P_{1}(0)-P_{2}(0)}=\exp \left(-\frac{t}{\tau_{R}}\right)
$$

as well as the pressure variations in each tank $(i=1,2)$

$$
P_{i=1,2}=P_{m} \pm\left(\frac{P_{1}(0)-P_{2}(0)}{2}\right) \exp \left(-\frac{t}{\tau_{R}}\right)
$$

Eq. 10 explains the symmetrical exponential relaxations observed in Fig. 4 for the gas pressures $P_{1}$ and $P_{2}$. The pressure relaxation kinetics is rigorously exponential in the isothermal regime and the associated characteristic time is given by:

$$
\tau_{R}=\frac{\mu V L}{2 A P_{m} K_{\mathrm{app}}}
$$

In $\$ 3.1$ it was noted that the measured relaxation time increases for decreasing pressure values. This result appeared to be paradoxical given that the slip effect increases for decreasing pressure values (at fixed temperature) leading to higher apparent permeability and theoretically shorter relaxation times. However, Eq. 11 shows that the mean pressure $P_{m}$ appears explicitly in the denominator of the $\tau_{R}$ expression: this factor is overwhelmingly greater than the slip effect.

When the variation in gas pressure in the tanks is isothermal, the apparent permeability can easily be determined from the semi-log plot of the pressure difference $\left(P_{1}(t)-P_{2}(t)\right) /\left(P_{1}(0)-P_{2}(0)\right)$ as a function of time. For example, Fig. 5 replots the data of Fig. 4 and it can be seen that the data values are aligned along a straight line characteristic of an isothermal regime. For this test, values of $\tau_{R}=11400 \mathrm{~s}$ and $K_{\text {app }}=1.32 \times 10^{-17} \mathrm{~m}^{2}$ are found. 


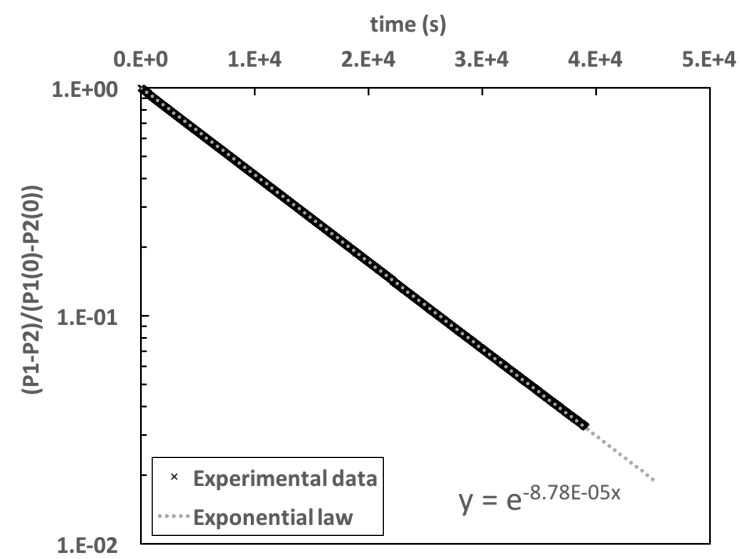

Fig. 5 Semi-log plot of the pressure difference $\left(P_{1}(t)-P_{2}(t)\right) /\left(P_{1}(0)-P_{2}(0)\right)$ as a function of time for a Norway spruce sample taken in the tangential direction at the mean pressure $P_{m}=299 \mathrm{mbar}$. The exponential fit leads to $\tau_{R}=11400 \mathrm{~s}$ and $K_{\text {app }}=1.32 \times 10^{-17} \mathrm{~m}^{2}$.

\subsubsection{Non-isothermal regime}

For track-etched membranes, the relaxation time $\tau_{R}$ is lower or even much lower than the gas conduction time $\tau_{\mathrm{cd}}$. In this case, the pressure relaxation in the tanks can no longer be considered to be isothermal over time. The gas in the high pressure (resp. low pressure) tank is considered to vary homogeneously and can be described solely by the pressure $P_{1}(t)$ and the temperature $T_{1}(t)$ (resp. $P_{2}(t)$ and $T_{2}(t)$ ). The tank walls remain at the chamber temperature $T_{0}$ since their thermal inertia is very high compared to the inertia of the gas enclosed in the set-up. The heat transfer between the gas and the tank is characterized by the conductance $\Gamma$. For the big tanks, $\Gamma$ was estimated to be about $2.1 \mathrm{~W} . \mathrm{K}^{-1}$ (Ai et al. 2017). Lastly, it was assumed that the flow in the sample is quasisteady and isothermal at the temperature $T_{1}(t)$ considering that the temperature decrease due to decompression is strictly counterbalanced by the viscous dissipation inside the porous medium (Figué, 1996).

The energy balance for the high-pressure tank is then given by:

$$
\frac{P_{1}(t) V}{R T_{1}(t)} C_{p g} \frac{d T_{1}(t)}{d t}=\frac{d P_{1}(t)}{d t} V+\Gamma\left(T_{0}-T_{1}(t)\right)
$$

where $C_{p g}$ is the molar heat capacity at constant gas pressure. The energy balance for the low pressure tank is thus:

$$
\frac{P_{2}(t) V}{R T_{2}(t)} C_{p g} \frac{d T_{2}(t)}{d t}=\frac{d P_{2}(t)}{d t} V+J(t) C_{p g}\left(T_{1}(t)-T_{2}(t)\right)+\Gamma\left(T_{0}-T_{2}(t)\right)
$$

The molar flow $J(t)$ can be expressed as a function of the variables associated with the high-pressure tank:

$$
J(t)=-\frac{V}{R T_{1}(t)} \frac{d P_{1}(t)}{d t}+\frac{P_{1}(t) V}{R} \frac{d T_{1}(t) / d t}{T_{1}^{2}(t)}
$$

and also as a function of the variables associated with the low-pressure tank:

$$
J(t)=\frac{V}{R T_{2}(t)} \frac{d P_{2}(t)}{d t}-\frac{P_{2}(t) V}{R} \frac{d T_{2}(t) / d t}{T_{2}^{2}(t)}
$$

The set of equations 6 and 12-15 fully describes the gas pressure variations in the set-up. Thus for each experimental test, the apparent permeability $K_{\text {app }}$ (associated with the mean pressure $\left.P_{m}=\left(P_{1}(0)+P_{2}(0)\right) / 2\right)$ may be determined by an inverse procedure: the pressure relaxations are calculated by solving the above system using the initial conditions of the test and a guessed permeability value. The calculated pressure changes are then compared to the experimental profiles. The objective function (to be minimized) is defined as the mean square difference between the simulated and measured pressure evolutions. 
Fig. 6 shows an example of experimental and associated calculated pressure relaxations for a $10 \mu \mathrm{m}$ track-etched membrane at $P_{m}=957$ mbar. The apparent permeability is equal to $K_{\text {app }}=1.1 \times 10^{-13} \mathrm{~m}^{2}$. The same value is obtained when non-isothermal conditions are assumed with a conductance $\Gamma$ tending towards zero which means that this configuration is close to an adiabatic system. In contrast, a significantly higher value, i.e. $K_{\text {app }}=$ $1.5 \times 10^{-13} \mathrm{~m}^{2}$, would have been obtained with the isothermal model (Eq. 9-11). The permeability values determined from an experiment on a $10 \mu \mathrm{m}$ membrane at $P_{m}=30$ mbar are: $K_{\text {app }}=4.2 \times 10^{-13} \mathrm{~m}^{2}$ with the non-isothermal model (with $\Gamma=2.1 \mathrm{~W} \cdot \mathrm{K}^{-1}$ ), $K_{\text {app }}=3.1 \times 10^{-13} \mathrm{~m}^{2}$ assuming an adiabatic system $(\Gamma=0)$ and $K_{\text {app }}=4.4 \times 10^{-13} \mathrm{~m}^{2}$ assuming isothermal conditions. It may therefore be concluded that the relaxation regime changes from adiabatic to isothermal as the relaxation time increases. These experimental results confirm the numerical simulations reported by Ai et al. (2017).

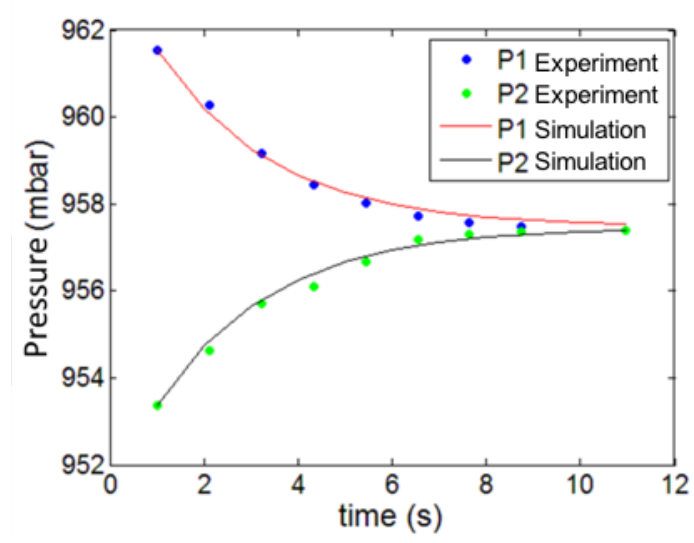

Fig. 6 Experimental and calculated pressure relaxations (assuming non-isothermal conditions) minimizing the objective function. Experimental data were obtained with a $10 \mu \mathrm{m}$ track-etched membrane at a mean pressure $P_{m}=957$ mbar. The apparent permeability is equal to $K_{\text {app }}=1.1 \times 10^{-13} \mathrm{~m}^{2}$.

\subsection{Klinkenberg plots}

A series of relaxation measurements was performed at different mean pressures for the $80 \mathrm{~nm}$ and $10 \mu \mathrm{m}$ tracketched membranes, and also for Norway spruce samples taken in the longitudinal and tangential directions (2 samples per direction of the wood $\log$ ). The experimental data obtained with the track-etched membranes were analyzed with the non-isothermal model whereas the data obtained with the Norway spruce samples were analyzed assuming isothermal conditions. The variations in the apparent permeability as a function of the reciprocal mean pressure are reported in Fig. 7.

The apparent permeability was found to increase as the mean pressure decreases: this is due to the gas slippage which increases with the Knudsen number Kn and becomes significant for $\mathrm{Kn}>0.01$. As the mean pressure decreases from 1000 mbar to 30 mbar, the Knudsen number in fact increases from 2 to 60 (resp. from $10^{-2}$ to $0.5)$ in the pores of the $80 \mathrm{~nm}$ (resp. $10 \mu \mathrm{m}$ ) track-etched membrane. For Norway spruce samples in the same pressure range, the Knudsen number varies from $10^{-2}$ to 0.4 in the latewood tracheids (calculated with the lumen radius measured by ESEM, i.e. $5.1 \mu \mathrm{m}$ ) and from 0.6 to 20 in the margo openings of the bordered pits (calculated with the mean value reported by Domec et al. (2006), i.e. $0.25 \mu \mathrm{m}$ ). 

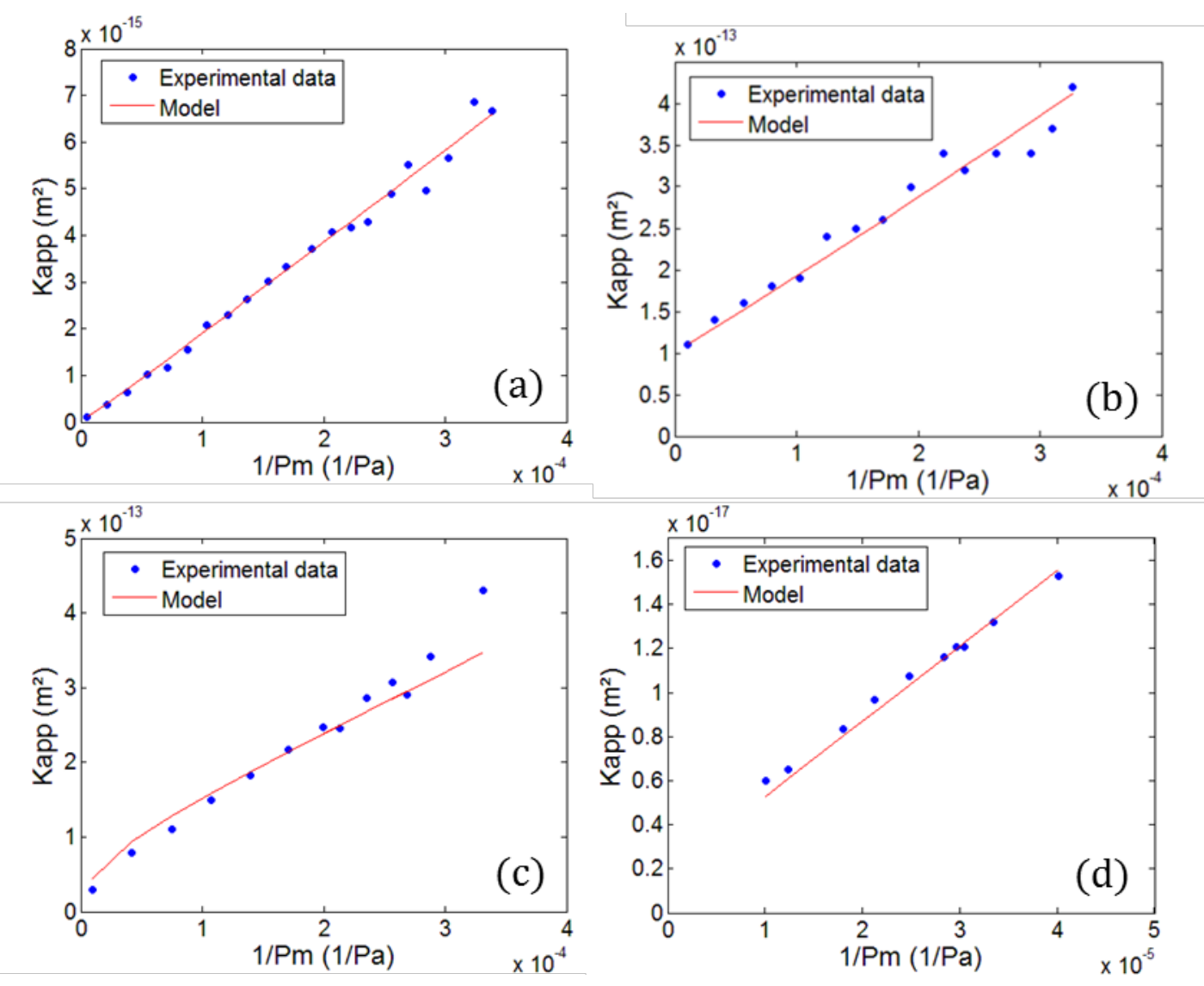

Fig. 7 Variations in the apparent permeability $K_{\text {app }}$ as a function of the reciprocal mean pressure $1 / P_{m}$ : (a) $80 \mathrm{~nm}$ track-etched membrane, (b) $10 \mu \mathrm{m}$ track-etched membrane, (c) Norway spruce sample taken in the longitudinal direction of the wood $\log$ (sample L1) and (d) Norway spruce sample taken in the tangential direction (sample T1): experimental data (dots) and calculated values (solid line)

For the $80 \mathrm{~nm}($ resp. $10 \mu \mathrm{m})$ track-etched membranes, the permeability varies from $1.1 \times 10^{-16} \mathrm{~m}^{2}$ to $6.9 \times$ $10^{-15} \mathrm{~m}^{2}$ (resp. from $1.1 \times 10^{-13} \mathrm{~m}^{2}$ to $4.2 \times 10^{-13} \mathrm{~m}^{2}$ ) when the mean pressure decreases from $1930 \mathrm{mbar}$ to $31 \mathrm{mbar}$ (resp. from $957 \mathrm{mbar}$ to $31 \mathrm{mbar}$ ). For the Norway spruce samples, the permeability in the longitudinal (resp. tangential) direction increases from $2.9 \times 10^{-14} \mathrm{~m}^{2}$ at $999 \mathrm{mbar}$ (resp. $6 \times 10^{-18} \mathrm{~m}^{2}$ at 993 mbar) to $4.3 \times 10^{-13} \mathrm{~m}^{2}$ at $30 \mathrm{mbar}$ (resp. $1.5 \times 10^{-17} \mathrm{~m}^{2}$ at $249 \mathrm{mbar}$ ). The intrinsic permeability $K_{\infty}$ was estimated by linear extrapolation of the data for a reciprocal mean pressure tending towards zero. A value of $K_{\infty}=$ $9.6 \times 10^{-14} \mathrm{~m}^{2}$ was obtained for the $10 \mu \mathrm{m}$ track-etched membrane to be compared with $7.9 \times 10^{-14} \mathrm{~m}^{2}$ estimated from Poiseuille's law in short capillaries (the pore density and the mean pore radius determined from the ESEM micrographs were used and the Couette correction factor (Bond 1921) was included to account for the end effects). The intrinsic permeability for the $80 \mathrm{~nm}$ membrane could not be estimated due to the absence of experimental data in the slip (and viscous) regime. For the Norway spruce sample taken in the longitudinal direction of the wood $\log$, a value of $K_{\infty}=2.3 \times 10^{-14} \mathrm{~m}^{2}$ was found for sample L1 (Fig. 7c) and $K_{\infty}=$ $2.65 \times 10^{-14} \mathrm{~m}^{2}$ for sample L2 (not shown), which is in very good agreement with the value reported by Tarmian and Perré (2009). In the tangential direction, values of $K_{\infty}=2.9 \times 10^{-18} \mathrm{~m}^{2}$ for sample T1 (Fig. 7d) and $K_{\infty}=2.7 \times 10^{-18} \mathrm{~m}^{2}$ for sample T2 (not shown) were obtained. The longitudinal to tangential permeability ratio in the Norway spruce samples is huge, i.e. about $10^{4}$, similar to the values reported by Comstock for spruce species (1970). This is a consequence of the strongly anisotropic organization of the wood tissue.

\section{Determination of pore-network parameters}

Pore-network models are synthetic representations of the structure of porous media. They are mesoscopic models. In terms of scale, this class of model lies between the microscopic approach, where the microgeometry of the porous medium is fully described, and the macroscopic approach, where the porous medium is described as an equivalent continuous medium with effective properties. In the present case, pore-networks typically consist of elementary building blocks such as pipes or orifices connected in series or in parallel. For a given porous 
medium, a suitable network must first be defined followed by selection of a set of appropriate building blocks. Usually network definition and block selection are based on side information provided, for instance, by literature (wood anatomy) or by the manufacturer (membrane specifications). The conductance of a building block is defined as the ratio of the volumetric flow rate traversing the block to the pressure difference between the block inlet and outlet. The overall conductance of the pore-network is deduced using an analogy between fluid flows and an electrical circuit (non-linear effects associated with contiguous margo openings are neglected for instance). In the present study, the free-parameters of the network (such as pore connectivity) and the building block characteristics (such as duct radius or orifice radius) were determined by minimizing the deviation between the experimental and simulated variations in apparent permeability.

\subsection{Track-etched polycarbonate membranes}

The membrane porosity is considered to consist of identical parallel cylindrical pores crossing the polycarbonate sheet perpendicularly. The gas flow in the pore is assumed to be isothermal and is represented by Beskok and Karniadakis's pipe model (1999) since it is valid over a wide range of Knudsen numbers. The following expression for the apparent membrane permeability is then obtained:

$$
K_{\mathrm{app}}=\frac{N \pi r^{4}}{8}(1+\alpha \mathrm{Kn})\left[1+4 \frac{2-\sigma}{\sigma} \frac{\mathrm{Kn}}{1-b \mathrm{Kn}}\right]
$$

The mean free path used in the Knudsen number $\mathrm{Kn}=\lambda / r$ is calculated according to Eq. 2 with the pressure set at $P_{m} . \quad \alpha$ is a function of Kn given by Beskok and Karniadakis (1999). $\sigma$ is the accommodation coefficient. According to Ewart et al. (2007) and Agrawal and Prabhu (2008), $\sigma$ lies typically between 0.7 and 1 (total accommodation) for flow through microchannels. $b$ is the so-called general slip coefficient. Beskok and Karniadakis determined that $b=-1$ for pipe flows assuming total accommodation.

The pore-network unknowns are the pore density $N$ and the mean pore radius $r$. Values for $N$ and $r$ were determined by minimizing the mean square difference between the permeability calculated with Eq. 16 and the experimental data (see Fig. 7). Unless indicated to the contrary, the values were determined assuming full accommodation $(\sigma=1)$. For the $80 \mathrm{~nm}$ membrane, values of $N=1.4 \times 10^{8}$ pores $/ \mathrm{cm}^{2}$ and $r=95 \mathrm{~nm}$ were found. Note that the discrepancy between the calculated values and those measured by ESEM is quite high (more than $100 \%$ for the pore radius) but the order of magnitude is correct. The value of $r$ was also calculated using the ESEM measured value of $N$ and this gave $r=58 \mathrm{~nm}$ which is in better agreement with the ESEM measurements. The same curves for $K_{\text {app }}$ versus $1 / P_{m}$ were obtained for either the calculated value of $N$ or the ESEM measured value of $N$. For the $10 \mu \mathrm{m}$ membrane, values of $N=9.9 \times 10^{4}$ pores $/ \mathrm{cm}^{2}$ and $r=4.1 \mu \mathrm{m}$ were found which are in good agreement with the ESEM measurements. The effect of the accommodation coefficient is significant since, for $\alpha=0.7$, the corresponding values were $N=2 \times 10^{4}$ pores $/ \mathrm{cm}^{2}$ and $r=6$ $\mu \mathrm{m}$.

A sensitivity analysis is needed in order to understand why both parameters can be reliably determined for the $10 \mu \mathrm{m}$ membrane, but only one for the $80 \mathrm{~nm}$ membrane. For this purpose, the sensitivity function $S_{u}$ was used, defined for $u=N$ and $u=r$ by:

$$
S_{u}=\frac{u}{K_{\mathrm{app}}} \frac{\partial K_{\mathrm{app}}}{\partial u}
$$

$K_{\text {app }}$ is here given by Eq. 16. The two functions $S_{r}$ and $S_{N}$ are plotted versus the Knudsen number Kn in Fig. 9. The value of $S_{r}$ decreases from 4 , when Kn is less than $10^{-2}$ (corresponding to the $r^{4}$-scaling of the viscous regime) to 3 when $\mathrm{Kn}$ is greater than 5 (corresponding to the $r^{3}$-scaling of the free molecular regime). The function $S_{N}$ is constant and equal to 1 according the $N^{1}$-scaling of Eq. 16. In the Kn-range investigated for the $80 \mathrm{~nm}$ membrane (displayed in green in Fig. 8), $S_{r}$ and $S_{N}$ essentially vary in the same way ( $S_{N}$ is a constant function and $S_{r}$ is almost constant over that Kn-range). Consequently, only one of the parameters $r$ and $N$ can be reliably determined. Conversely, in the Kn-range investigated for the $10 \mu \mathrm{m}$ membrane (displayed in purple in Fig. 8), the values of $S_{r}$ and $S_{N}$ do not vary in the same way and the simultaneous reliable determination of $r$ and $N$ is thus possible. This sensitivity analysis shows that the values of the two parameters are effectively independent only if the flow regime changes significantly over the investigated pressure range (the gas flow soon shifts from transition regime to slip regime). 
Even so, uncertainties still remain regarding the calculated parameters related to (i) the isothermal assumption for the gas flow in the pores, (ii) the pore aspect ratio $L / r$ which is not high enough for Beskok and Karniadakis's pipe model to be strictly applied (for the $10 \mu \mathrm{m}$ membrane) and (iii) the total accommodation assumption.

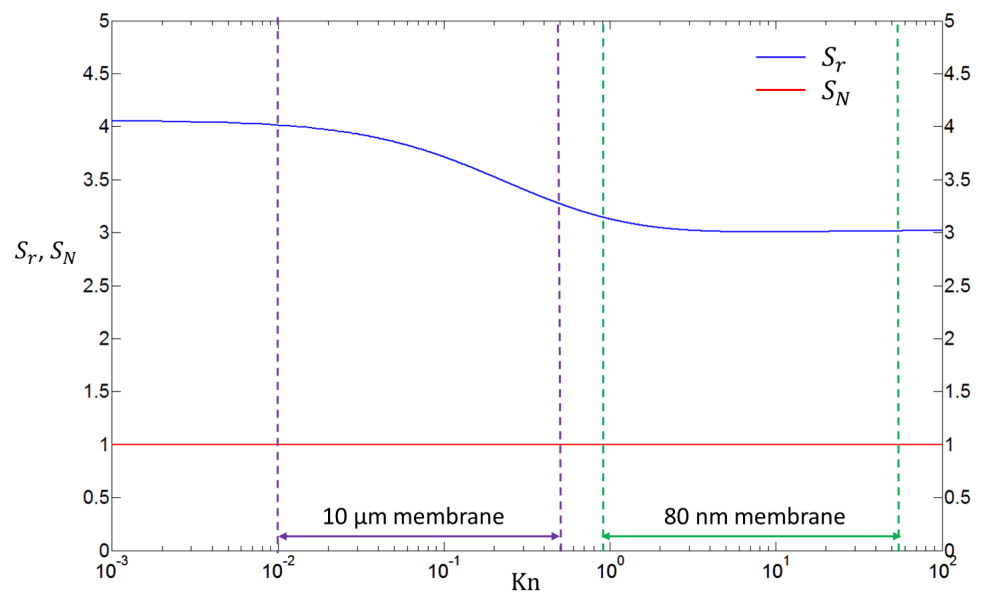

Fig. 8 Variations in $S_{r}$ and $S_{N}$ as a function of the Knudsen number: the Kn-range experimentally investigated for the $80 \mathrm{~nm}$ membrane (resp. $10 \mu \mathrm{m}$ membrane) is represented in green (resp. purple)

\subsection{Norway spruce}

Comstock's model (1970) was chosen as a suitable tool for modeling gas permeability in Norway spruce. This model relies on basic knowledge in wood anatomy and approximates softwood structure as follows: (i) tracheids are long rectangular boxes with non-perforated ends, (ii) the bordered pits are uniformly distributed on the radial walls of the tracheids and (iii) on average, tracheids overlap adjacent tracheids over half of their length. A scheme of this structure is presented in Fig. 9. A comparison between Fig. 9 and the micrographs of Fig.1 shows that Comstock's assumptions are actually relevant.

The pits are mainly aspired in earlywood. Consequently, earlywood has a much lower gas permeability than latewood (Phillips 1933, Almeida et al. 2008). In this model, earlywood is therefore considered to be totally impermeable. $\phi$ is the latewood volume fraction. $h$ is the tracheid length. $w$ and $l$ are the radial and tangential dimensions (including the cell wall) of the latewood tracheids.

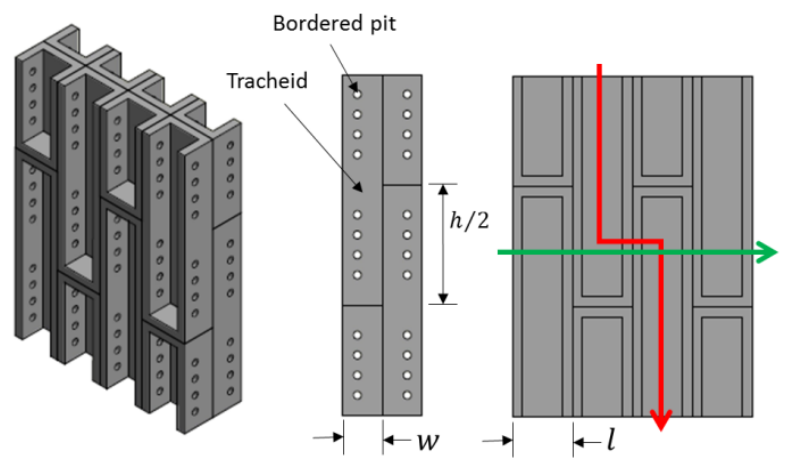

Fig. 9 Comstock's model (1970) of softwood structure: perspective view of a set of tracheids (left), view in the LT plane (middle), view in the LR plane (left): the red arrow indicates the gas flow path in the longitudinal direction and the green arrow indicates the flow path in the tangential direction

The bordered pits are taken into account via the margo openings since it is mainly these openings that govern the gas flow. The proposed model for gas flow in the tracheids is based on Beskok and Karniadakis's pipe model while the Borisov et al. (1973) orifice model is used for gas flow in the margo openings. Modeling the margo openings as a set of isolated orifices may appear to be a massive simplification given that the margo is a $2 \mathrm{D}$ - 
network of nanofibers. However, a careful observation of this network confirms that a small number of openings are much larger than others, typically 10 to 12 openings (Comstock and Côté, 1968). The remaining parts of the margo which have a very tight network, are unlikely to make a significant contribution to the gas flow.

The longitudinal conductance $\kappa_{1}$ of half a tracheid is given by:

$$
\kappa_{1}=\frac{\pi r_{1}^{4}}{4 \mu h}\left(1+\alpha \mathrm{Kn}_{1}\right)\left[1+4 \frac{2-\sigma}{\sigma} \frac{\mathrm{Kn}_{1}}{1-b \mathrm{Kn}_{1}}\right]
$$

where $r_{1}$ is the tracheid lumen equivalent radius, $\mathrm{Kn}_{1}=\lambda / r_{1}$ the Knudsen number associated with the gas flow in the tracheids and $\lambda$ calculated at $P_{m}$.

In adddition, the conductance of an opening is given by:

$$
\kappa_{2}=\frac{5 \pi r_{2}^{3}}{16 \mu} \mathrm{Kn}_{2} \xi_{P}^{o r}
$$

where $r_{2}$ is the radius of the openings in the margo, $\mathrm{Kn}_{2}=\lambda / r_{2}=\left(r_{2} / r_{1}\right) \mathrm{Kn}_{1}$ is the Knudsen number associated with the gas flow through an opening, $\lambda$ is calculated at $P_{m}$ and $\xi_{P}^{o r}$ is a coefficient which depends on $\mathrm{Kn}_{2}$ (Sharipov and Seleznev, 1998).
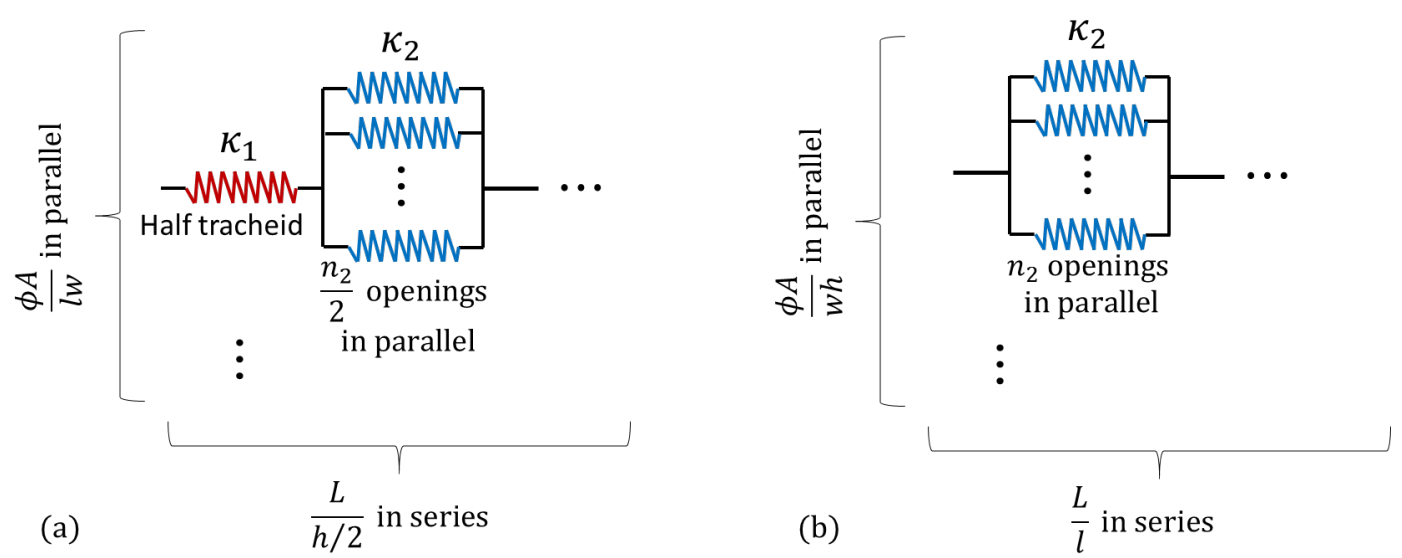

Fig. 10 Conductance network representing gas permeation in the longitudinal (a) and tangential (b) directions of Norway spruce samples: the half tracheid conductance is displayed in red and the conductance of an opening in a margo in blue.

Gas permeation in the longitudinal direction of Comstock's structure can be represented by the conductance network of Fig. 10a. Thus, for a permeation test in the longitudinal direction, the gas volume flow rate through the sample is given by:

$$
Q_{\text {long }}=\left[\left(\kappa_{1}^{-1}+\left(\frac{n_{2}}{2} \kappa_{2}\right)^{-1}\right) \frac{L}{h / 2}\right]^{-1} \frac{A \phi}{w l} \Delta P
$$

where $n_{2}$ is the number of margo openings per tracheid. According to Fig. 10b the gas volume flow rate in the tangential direction is given by:

$$
Q_{\text {tang }}=n_{2} \kappa_{2} \frac{l}{L} \frac{A \phi}{w h} \Delta P
$$

From Eq. 18-21 the limit of the longitudinal to tangential permeability ratio for a Knudsen number tending towards zero can be expressed as:

$$
\lim _{\mathrm{Kn}_{1} \rightarrow 0} \frac{K_{\text {long }}}{K_{\text {tang }}} \cong \frac{h^{2}}{4 w^{2}}
$$

Since the tracheid length $h$ varies between $2.8 \mathrm{~mm}$ and $4.29 \mathrm{~mm}$ for mature Norway spruce (Brändström 2001), the value of $h$ will be about $3.5 \mathrm{~mm}$. Moreover, ESEM measurements have shown that $w=17 \mu \mathrm{m}$ in latewood. 
A value of $h^{2} /\left(4 w^{2}\right) \cong 1.1 \times 10^{4}$ is then obtain which is in reasonable agreement with the ratio of intrinsic permeabilities given by our measurements, in the order of $8 \times 10^{3}$ to $10^{4}$. This confirms that Norway spruce structure is well described by Comstock's model.

Three unknowns are considered for the determination: i) the volume fraction $\phi$ of latewood, ii) the number $n_{2}$ of margo openings per tracheid in latewood and iii) the effective opening radius $r_{2}$. The tracheid length $h$ was fixed at $3.5 \mathrm{~mm}$. The values of $w, l$ and $r_{1}$ were those determined by ESEM observations for latewood. The determination was performed simultaneously in the longitudinal and tangential directions assuming full accommodation. It should be noted that since the wood samples were cut from the same plank of a "resonance spruce", it may be assumed that the samples have the same latewood fraction $\phi$. The values determined on samples L1 and T1 (resp. L2 and T2) are $\phi=0.26, r_{2}=0.12 \mu \mathrm{m}$ and $n_{2}=90$ (resp. $\phi=0.30, r_{2}=0.10 \mu \mathrm{m}$ and $n_{2}=56$ ).

The low value of $r_{2}$ confirms that softwood permeability (in the longitudinal and tangential directions) is governed by the margo openings rather than by the pit apertures. Although Norway spruce is well documented (Lindström 1997, Brändstrom 2001, Trtik et al. 2007), no thorough study of Norway Spruce bordered pits could be found in the literature. However, a study focusing on Douglas fir was found (Domec et al. 2006). According to pit micrographs (showing the torus and the margo), Norway spruce pits (Barlow and Woodhouse 1990) look very similar those found in Douglas fir (Domec et al. 2006). Domec et al. measured the characteristics of bordered pits from scanning electron micrographs and reported a mean opening radius of $0.22 \mu \mathrm{m}$ to $0.31 \mu \mathrm{m}$ in latewood. The opening radius values determined in the present study are in good agreement with the results found by Domec et al. (even though the calculated values are lower than those measured by Domec et al.). The results are also in very good agreement with the value (about $0.15 \mu \mathrm{m}$ ) determined by Petty on Sitka spruce in the longitudinal direction. Note that for the transport of water in wood, Hacke et al. (2004) similarly found that the equivalent opening diameter (determining the hydraulic resistance) was lower than the "real" geometric diameter of the opening, i.e. the equivalent diameter was approximately $63 \%$ of the geometric diameter.

According to Tjoelker et al. (2007), the number of pits per tracheid varies between 70 and 210 for Norway spruce. The lower value typically corresponds to the number of pits per tracheid in latewood, which has only one line of tracheids. Domec et al. data (2006) also show that the number of openings in the margo of a bordered pit is of the order of $10^{2}$. However, from a careful observation of the margo, it is clear that, of this number, the quantity of large openings, mainly involved in the gas flow, is much lower, probably of the order of 10-12 (Comstock and Côté, 1968). Since the number of pit openings per tracheid was calculated to be about 50 to 100 , it is to be expected that about 10 bordered pits per tracheid will be flow conducting in the latewood of the samples tested. The proportion of aspired pits in latewood should therefore be of the order of $85 \%$. This value agrees quite well with data found in the literature: Bao et al. (2001) reports that the proportion of aspired pits in heartwood samples of Chinese yezo spruce after air-drying is equal to $86.3 \%$ in latewood and $97.5 \%$ in earlywood.

Lastly, the calculated latewood fraction is greater than the value estimated by binocular loupe, i.e. about 0.16 .

As in section 4.1, the variations in $S_{u}$ were calculated as a function of $\mathrm{Kn}$ for $u=\phi, r_{2}$ and $n_{2} . S_{u}$ is calculated for the apparent permeability in the longitudinal direction (Eq. 20) and in the tangential direction (Eq. 21). The corresponding curves are displayed in the electronic supplementary material (see Fig. B1 and Fig. B2). The variations in $S_{\phi}, S_{r_{2}}$ and $S_{n_{2}}$ values for the apparent permeability in the longitudinal direction would appear to be distinct in the investigated Kn-range: the values of $\phi, r_{2}$ and $n_{2}$ can in fact be determined. However, $S_{\phi}$ and $S_{n_{2}}$ values for the apparent permeability in the tangential direction vary in the same way: both are constant functions that are consistent with the fact that $\phi$ and $n_{2}$ are found in Eq. 21 in the form of the product $\phi n_{2}$. It is thus impossible to separate the effect of $\phi$ from the effect of $n_{2}$ from tangential data alone.

These results show that the determination from combined longitudinal and tangential data is more reliable for the opening radius $r_{2}$ than for the opening number $n_{2}$ and the latewood fraction $\phi$. Even with the longitudinal data, it proved difficult to separate the effect of $\phi$ from that of $n_{2}$. This difficulty is explained by the dependence of Eq. 20 on $\phi$ and $n_{2}$ which degenerates into a dependence on just the product $\phi n_{2}$ for a Knudsen number tending towards zero. Assuming that the determination of the product $\phi n_{2}$ is more reliable, a value of $n_{2}$ may be deduced that is consistent with the latewood fraction estimated by binocular loupe $\phi=0.16$. Since it was found that $\phi n_{2} \approx 20$, a value of $n_{2} \approx 125$ is obtained. This value does not significantly change the percentage of aspirated pits in latewood. 


\section{Conclusion}

The present work is based on a method originally developed by Petty in the late sixties to determine morphological parameters of dry wood from gas permeability measurements. New datasets were first generated using an improved experimental device capable of measuring the apparent gas permeability over a wide range of permeability values (from $10^{-10}$ to $10^{-18} \mathrm{~m}^{2}$ ) and a wide range of mean gas pressures (from 2 bar down to 35 mbar). In this approach, the transient character of the experiments is rigorously taken into account in the data analysis and the apparent permeability is determined from the pressure relaxation kinetics by inverse analysis. Morphological parameters were then identified from the variations in apparent permeability as a function of the mean pressure using a pore network model. The choice of the network and its elementary building blocks is based on side information provided, for instance, by literature (wood anatomy) or by the manufacturer (membrane specifications).

This approach was first applied to track-etched membranes characterized by well-defined cylindrical pores. When flow regime changes occurred in the investigated pressure range, the pore density and the mean pore radius of the membrane could be determined and these values were found to be in good agreement with ESEM observations.

Lastly, Norway spruce specimens taken in the tangential and longitudinal directions of the wood log were investigated. The mean radius of the pores, i.e. openings in the margo of the bordered pits, governing wood permeability in the longitudinal and tangential directions was determined and was in good agreement with the data reported in literature. The mean volume fraction of latewood (i.e. the permeable fraction of air-dried wood) and the mean number of openings per tracheid were also determined. The proportion of aspirated pits is in reasonable agreement with literature data whereas the latewood volume fraction is overestimated (0.26 against 0.16) compared to the value estimated by binocular loupe. It is in fact difficult to separate the effects of latewood fraction and number of openings per tracheid since the permeability value depends essentially on the product of these two parameters.

More generally, the present approach can be used to characterize the micro-morphology of a variety of porous materials such as rocks, soils, building materials or food materials, as long as the porosity concerned is open and interconnected and can be properly described by a pore network model (Mehmani et al. 2013, Esveld et al. 2012).

\section{Electronic supplementary material}

See the electronic supplementary material for details on: A, Experimental pressure relaxation kinetics ; B, Norway spruce and the sensitivity of the apparent permeability to the parameters $\phi, r_{2}$ and $n_{2}$.

\section{References}

Adzumi H (1937) On the flow of gases through a porous wall. Bull Chem Soc Japan 12:304-312

Agoua E, Perré P (2010) Mass transfer in wood: Identification of structural parameters from diffusivity and permeability measurements. Journal of porous media, 13:1017-1024

Agrawal A, Prabhu SV (2008) Survey on measurement of tangential momentum accommodation coefficient. J Vac Sci Technol A 26:634-645

Ai W, Duval H, Pierre F, Perré P (2017) A novel device to measure gaseous permeability over a wide range of pressure: characterization of slip flow for various wood species and wood-based materials. Holzforschung 71: 147-162

Almeida G, Leclerc S, Perre P (2008) NMR imaging of fluid pathways during drainage of softwood in a pressure membrane chamber. International Journal of Multiphase Flow 34:312-321

ASTM F2450-10 (2010) Standard Guide for Assessing Microstructure of Polymeric Scaffolds for Use in Tissue Engineered Medical Products, Book of Standards Volume 13.01, ASTM International, West Conshohocken, PA

Bao FC, Lu J, Zhao Y (2001) Effect of bordered pit torus position on permeability in Chinese yezo spruce. Wood Fiber Science 33:193-199

Barlow CY, Woodhouse J (1990) Bordered pits in spruce from old Italian violins. Journal of Microscopy 160:203-211

Beskok A, Trimmer W, Karniadakis GE (1996) Rarefaction and compressibility effects in gas microflows. J. Fluids Eng., 118 :448-456 
Beskok A, Karniadakis GE (1999) A model for flows in channels, pipes, and ducts at micro and nano scales. Microscale Thermophys Eng 3: 43-77

Bird GA (1994) Molecular Gas Dynamics and the Direct Simulation of Gas Flows. Clarendon Press, Oxford

Bond WN (1921) Viscosity Determination by Means of Orifices and Short Tubes. Proc Phys Soc London 34: 139-144

Borisov SF, Neadachin IG, Porodnov BT, Suetin PE (1973) Stream of rarefied gases through an opening at small pressure drops. Zhurnal Tekhnicheskoi Fiziki 43:1735-1739

Brändström J (2001) Micro- and ultrastructural aspects of Norway spruce tracheids: a review. IAWA Journal 22:333-353

Brenner H (2005) Kinematics of volume transport. Phys A 349:11-59

Civan F (2010) Effective correlation of apparent gas permeability in tight porous media. Transp Porous Med $82: 375-384$

Comstock GL, Côté WA (1968) Factors affecting permeability and pit aspiration in coniferous sapwood. Wood Science and Technology 2:279-291

Comstock GL (1970) Directional permeability of softwoods. Wood Fiber Sci 1:283-289

Dixon MA, Joly J (1894) On the ascent of sap. Philosophical Transactions of the Royal Society London, Series B 186: 563-576

Domec JC, Lachenbruch B, Meinzer FC (2006) Bordered pit structure and function determine spatial patterns of air-seeding thresholds in xylem of Douglas-fir (Pseudotsuga menziesii; Pinaceae) trees. American Journal of Botany 93: 1588-1600

Esveld DC, van der Sman RGM, van Dalen G, van Duynhoven JPM, Meinders MBJ (2012) Effect of morphology on water sorption in cellular solid foods. Part I: Pore scale network model. Journal of Food Engineering 109:301-310

Ewart T, Perrier P, Graur I, Méolans G (2007) Tangential momentum accommodation in microtube. Microfluid Nanofluid 3:689-695

Figué JF (1996) Modélisation numérique des écoulements compressibles en milieu poreux - Application à la détente de Joule-Thomson. Dissertation, Université de Bordeaux, France

Florence FA, Rushing JA, Newsham KE, Blasingame TA (2007) Improved permeability prediction relations for low permeability sands. Paper SPE 107954 presented at tha 2007 SPE Rocky Mountain Oil and Gas Technology Symposium, Denver, Colrado, USA, 16-18 April 2007

Hacke UG, Sperry JS, Pittermann J (2004) Analysis of circular bordered pit function II. Gymnosperm tracheids with torus-margo pit membranes. American Journal of Botany 91:386-400

Hirschfelder JO, Curtiss CF, Bird RB (1954) Molecular theory of gases and liquids. John Wiley and Sons Inc., New York

Karniadakis GE, Beskok A, Aluru N (2005) Microflows and nanoflows: fundamentals and simulation. SpringerVerlag, New York

Klinkenberg LJ (1941) The permeability of porous media to liquids and gases. Paper presented at the API 11 th Mid-Year Meeting, Tulsa, OK, May 1941; In: API Drilling and Production Practice, American Petroleum Institute, Whashington, DC, 200-213

Lindström H (1997) Fiber length, tracheid diameter, and latewood percentage in Norway spruce: development from pith outwards. Wood and Fiber Science 29:21-34

Liu H, Xu K, Zhu T, Ye W (2012) Multiple temperature kinetic model and its applications to micro-scale gas flows. Computers \& Fluids $67: 115-122$

Lu J, Avramidis S (1999) Non-Darcian air flow in wood. Part 3. Molecular slip flow. Holzforschung 53:85-92

Lv Q, Wang E, Liu X, Wang S (2014) Determining the intrinsic permeability of tight porous media based on bivelocity hydrdynetics. Microfluid Nanofluid 16:841-848

Maxwell JC (1879) On stresses in rarified gases arising from inequalities of temperature. Phil Trans Roy Soc 170:231-256

Mehmani A, Prodanovic M, Javadpour F (2013) Multiscale, multiphysics network modeling of shale matrix gas flows. Transp Porous Med 99:377-390

Petty JA (1970) Permeability and structure of the wood of Sitka spruce. Proc Roy Soc Lond B 175: 149-166 
Petty JA, Preston RD (1969) The dimensions and number of pit membrane pores in conifer wood. Proc Roy Soc Lond B 172:137-151

Phillips EWJ (1933) Movement of the pit membrane in coniferous woods, with special reference to preservative treatment. Forestry 7:109-120

Rasband WS, ImageJ, U. S. National Institutes of Health, Bethesda, Maryland, USA, http://imagej.nih.gov/ij/, 1997-2014.

Rouquerol J, Avnir D, Fairbridge CW, Everett DH, Haynes JH, Pernicone N, Ramsay JDF, Sing KSW, Unger KK (1994) Recommendations for the characterization of porous solids. Pure and Applied Chemistry 66:17391758

Scholander PF, Hammel HT, Bradstreet EA, Hemmingsen EA (1965) Sap pressure in vascular plants. Science 148:339-346

Sebastian LP, Côté WA, Skaar C (1965) Relationship of gas phase permeability to ultrastructure of white spruce wood. Forest Product J 15:394-404

Sharipov F, Seleznev V (1998) Data on internal rarefied gas flows. J Phys Chem Ref Data 27:657-706

Siau JF (1995) Wood: Influence of moisture on physical properties, Department of Wood Science and Forest Products, Virginia Polytechnic Institute and State University, Keene

Tarmian A, Perré P (2009) Air permeability in longitudinal and radial direction of reaction wood as compared to normal wood: compression wood of Picea abies and tension wood of Fagus sylvatica. Holzforchung 63:352-356

Tjoelker MG, Boratynski A, Bugala W (2007) Biology and Ecology of Norway Spruce, Springer, Dordrecht

Trtik P, Dual J, Keunecke D, Mannes D, Niemz P, Stähli P, Kaestner A, Groso A, Stampanoni M (2007) 3D imaging of microstructure of spruce wood. Journal of Structural Biology 159:46-55

Zhang WM, Meng G, Wei X (2012) A review on slip models for gas microflows. Microfluid Nanofluid 13:845882

Zhu T, Ye W (2010) Theoretical and numerical studies of noncontinuum gas-phase heat conduction in micro/nano devices. Numerical Heat Transfer, Part B: Fundamentals 57:203-226 